\title{
Strategic Classification from Revealed Preferences
}

\author{
JINSHUO DONG, University of Pennsylvania \\ AARON ROTH, University of Pennsylvania \\ ZACHARY SCHUTZMAN, University of Pennsylvania \\ BO WAGGONER, University of Pennsylvania \\ ZHIWEI STEVEN WU, Microsoft Research
}

\begin{abstract}
We study an online linear classification problem in which the data is generated by strategic agents who manipulate their features in an effort to change the classification outcome. In rounds, the learner deploys a classifier, then an adversarially chosen agent arrives and possibly manipulates her features to optimally respond to the learner's choice of classifier. The learner has no knowledge of the agents' utility functions or "real" features, which may vary widely across agents. Instead, the learner is only able to observe their "revealed preferences", i.e., the manipulated feature vectors they provide. For a broad family of agent cost functions, we give a computationally efficient learning algorithm that is able to obtain diminishing "Stackelberg regret" - a form of policy regret that guarantees that the learner is realizing loss nearly as small as that of the best classifier in hindsight, even allowing for the fact that agents would have best-responded differently to the optimal classifier.
\end{abstract}

Full Paper: https://arxiv.org/abs/1710.07887

CCS Concepts: • Applied computing $\rightarrow$ Electronic commerce; Economics;

Additional Key Words and Phrases: strategic agents; online learning; Stackelberg regret; revealed preferences; strategic classification

\section{INTRODUCTION}

Machine learning is typically studied under the assumption that the data distribution a classifier is deployed on is the same as the data distribution it was trained on. However, the outputs of many classification and regression problems are used to make decisions about human beings, in settings such as determining whether an individual will receive a loan, be hired, be admitted to college, or whether their email will pass through a spam filter. In these settings, the individuals have a vested interest in the outcome, and so the data generating process is better modeled as part of a strategic game in which individuals edit their data before sending it to the classifier to increase the likelihood of a particular desired outcome. Tax evaders may carefully craft their tax returns to decrease the likelihood of an audit; home buyers may strategically sign up for more credit cards in an effort to increase their credit score; email spammers may modify their emails in order to evade existing filters. In each of these settings, the individuals have a natural objective that they want to maximize - they want to increase their probability of being (say) positively classified. However,

Authors' addresses: Jinshuo Dong, University of Pennsylvania, Philadelphia, PA, djs.pku@gmail.com; Aaron Roth, University of Pennsylvania, Philadelphia, PA, aaroth@cis.upenn.edu; Zachary Schutzman, University of Pennsylvania, Philadelphia, PA, ianzach@seas.upenn.edu; Bo Waggoner, University of Pennsylvania, Philadelphia, PA, bwag@seas.upenn.edu; Zhiwei Steven Wu, Microsoft Research, New York, NY, steven7woo@gmail.com.

Permission to make digital or hard copies of all or part of this work for personal or classroom use is granted without fee provided that copies are not made or distributed for profit or commercial advantage and that copies bear this notice and the full citation on the first page. Copyrights for components of this work owned by others than the author(s) must be honored. Abstracting with credit is permitted. To copy otherwise, or republish, to post on servers or to redistribute to lists, requires prior specific permission and/or a fee. Request permissions from permissions@acm.org.

(C) 2018 Copyright held by the owner/author(s). Publication rights licensed to ACM.

ACM EC'18, fune 18-22, 2018, Ithaca, NY, USA. ACM ISBN 978-1-4503-5829-3/18/06 ..\$15.00

https://doi.org/10.1145/3219166.3219193 
they also incur a cost from performing these manipulations (tax evaders may have to pay some tax to avoid an audit, and email spammers must balance their ability to evade spam filters with their original goal in crafting email text). These costs can be naturally modeled as the distance between the "true" features $x$ of the individual and the manipulated features $\hat{x}$ that he ends up sending, according to some measure. In settings of this sort, learning can be viewed as a game between a learner and the set of individuals who generate the data, and the goal of the learner is to compute an equilibrium strategy of the game that maximizes her utility.

The relevant notion of equilibrium depends on the order of information revelation in the game. Frequently, the learner will first deploy her classifier, and then the data generating players (agents) will get to craft their data with knowledge of the learner's classifier. In such settings, the learner should seek to play a classifier corresponding to a Stackelberg equilibrium of the game; she should deploy the classifier that minimizes her error after the agents are given an opportunity to bestrespond to the learner's classifier. This is the approach taken by the most closely related prior work: [8] and [14]. Both of these papers consider a one-shot game and study how to compute the Stackelberg equilibria of this game. To do this, they necessarily assume that the learner has (almost) full knowledge of the agents' utility functions; in particular, it is assumed that the learner has access to the "true" distribution of agent features (before manipulation), and that the costs experienced by the agents for manipulating their data are the same for all agents and known to the learner. ${ }^{1}$

The primary point of departure in our work is to study the strategic classification problem when the learner does not know the utility functions of the agents: neither their true features $x$, nor the cost they experience for manipulation (which we allow to differ for each agent). In this setting, it no longer makes sense to study learning as a one-shot game; we cannot compute an equilibrium when the utility functions of the agents are unknown. Instead, we model the learning process as an iterative, online procedure. In each round $t=1,2, \ldots T$, the learner proposes a classifier $\beta_{t}$. Then, an agent arrives, with an unknown set of $d$ "true" features $x_{t} \in \mathbb{R}^{d}$, and an unknown cost function $d_{t}: \mathbb{R}^{d} \times \mathbb{R}^{d} \rightarrow \mathbb{R}$ for manipulation. The learner observes only the manipulated set of features $\hat{x}_{t} \in \mathbb{R}^{d}$, which represents the agent's best response to $\beta_{t}$. After classification, the learner observes the agent's true label $y_{t}$, and suffers the corresponding loss. Crucially, the learner never gets to observe either $x_{t}$ or $d_{t}$, and we do not even assume that these are drawn from a distribution (they may be adversarially chosen). The only access that the learner has to these parameters is via the revealed preferences of the agents - the learner gets to observe the actions of the agents, which are the result of optimizing their unknown utility functions. The learner must use this information to improve her classifier.

We measure the performance of our algorithms via a quantity that we call Stackelberg regret: informally, by comparing the average loss of the learner to the loss she could have experienced with the best fixed classifier in hindsight, taking into account that the agents would have best-responded differently had she used a different classifier. If the learner were in fact interacting with the same agent repeatedly, or if the agents $\left(x_{t}, d_{t}\right)$ were drawn from a fixed distribution, then the guarantee of diminishing Stackelberg regret would imply the convergence to a Stackelberg equilibrium of the corresponding one-shot game. However, Stackelberg regret is more general, and applies even in settings in which the agents are adversarially chosen.

We add one further twist. Previous work on strategic classification has typically assumed that all agents are strategic. However, the equilibrium solutions that result from this assumption may be undesirable. For example, in a spam classification setting, the Stackelberg-optimal classifier may

\footnotetext{
${ }^{1}$ Brückner and Scheffer [8] model a single data generation player who manipulates the data distribution, and experiences cost equal to the squared $\ell_{2}$ distance of his manipulation. Hardt et al. [14] study a model in which each agent can independently manipulate his own data point, but assume that all agents have the same separable cost function, known to the learner.
} 
attain its optimal accuracy only if all agents, even legitimate (non-spam) senders, actively seek to manipulate their emails to avoid the spam filter. In these settings, it would be more desirable to compute a classifier that was optimal under the assumption that spammers would attempt to manipulate their emails in order to game the classifier, but that legitimate senders would not manipulate their emails. To capture this nuance, in our model, only agents whose true label $y_{t}=-1$ (e.g. spammers) are strategic, and agents for whom $y_{t}=1$ are non-strategic.

\subsection{Our Results and Techniques}

The problem that the learner must solve is a bi-level optimization problem in which the objective of the inner layer (the agents' maximization problem) is unknown. Even with full information, bi-level optimization problems are often NP-hard. As a first step in our solution, we seek to identify conditions under which the learner's optimization problem is convex. Under these conditions, computing an optimal solution would be tractable in the full information setting. The remaining difficulty is solving the optimization problem in our limited feedback model.

We study learners who deploy linear classifiers $\beta_{t}$, and consider two natural learner loss functions: logistic loss (corresponding to logistic regression) and hinge loss (corresponding to support vector machines). The agents in our model are parameterized by target feature vectors $x_{t}$ and cost functions $d_{t}$, and will play the modified feature vector $\hat{x}_{t}\left(\beta_{t}\right)$ that maximizes their utility given $\beta_{t}$. We model agent utility functions as $u_{t}(\hat{x}, \beta)=\langle\beta, \hat{x}\rangle-d_{t}\left(x_{t}, \hat{x}_{t}\right)$. Using tools from convex analysis, we give general conditions on the cost functions $d_{t}$ that suffice to make the learner's objective convex, for both logistic and hinge loss, for all $x_{t}$. These conditions are satisfied by (among other classes of cost functions) any squared Mahalanobis distance and, more generally, by any norm-induced metric raised to a power greater than one.

Finally, we turn to the learner's optimization problem. Once we have derived conditions under which the learner's optimization problem is convex, we can achieve quickly diminishing Stackelberg regret with any algorithm for online bandit (i.e. zeroth order) optimization that works for adversarialy chosen loss functions. However, we observe that when some of the agents are non-strategic (e.g. the non-spammers), there is additional structure that we can exploit. In particular, on rounds for which the agent is non-strategic, the learner can also derive gradients for her loss function, in contrast to rounds on which the agent is strategic, where she only has access to zeroth-order feedback. To take advantage of this, we analyze a variant of the bandit convex optimization algorithm of [12] which can make use of both kinds of feedback. The regret bound we obtain interpolates between the bound of [12], obtained when all agents are strategic, and the regret bound of online gradient descent [24], obtained when no agents are strategic, as a function of the proportion of the observed agents which were strategic.

\subsection{Further Related Work}

In the adversarial or strategic learning literature, the most closely related works are [8] and [14], as discussed above, which also consider notions of Stackelberg equilibrium, and make other similar modelling choices. [18] also model adversarial learning as a Stackelberg game. Other works in this line model the learning problem as a purely adversarial (zero sum) game as in [11] for which the appropriate solution concept is a minmax equilibrium, study the simultaneous move equilibria of non-zero sum games as in [6,7], and study the Bayes-Nash equilibria of incomplete information games given Bayesian priors on the player types as in [13]. Common to all of these works is the assumption that the learner either has full knowledge of the data generator's utility functions (when Nash and Stackelberg equilibria are computed), or else knowledge of a prior distribution (when Bayes-Nash equilibria are computed). The point of departure of the current paper is to 
assume that the learner does not have this knowledge, and instead only has the power to observe agent decisions in response to deployed learners.

There is a parallel literature in machine learning and algorithmic game theory focusing on the problem of learning from revealed preferences - which corresponds to learning from the choices that agents make when optimizing their (unknown) utility functions in response to some decision of the learner as in [4]. This literature has primarily focused on how buyers with unknown combinatorial valuation functions make purchases in response to prices. Learning problems studied in this literature include learning to predict what purchase decisions a buyer will make in response to a set of prices drawn from an unknown distribution as in [3,23], finding prices that will maximize profit or welfare after buyers best respond as in [1,20,21], and generalizations of these problems as in [15]. We study the problem of strategic classification with this sort of "revealed preferences" feedback.

Finally, Stackelberg games are studied extensively in the "security games" literature: see [22] for an overview. Most closely related to this paper is the work of [2], who develop no-regret algorithms for certain kinds of security games when "attackers" arrive online, using a notion of regret that is equivalent to the "Stackelberg regret" that we consider. This work is similar in motivation to ours; its goal is to give algorithms to compute equilibrium-like strategies for the "defender" without assuming that he has an unrealistic amount of knowledge about his opponents' utility functions. Technically, the work is quite different, since we are operating in very different settings. In particular, [2] are primarily interested in information theoretic bounds, and do not give computationally efficient algorithms (since in general, solving Stackelberg security games is NP hard - see [16].)

\section{MODEL AND PRELIMINARIES}

We study a sequential binary classification problem in which a learner wishes to classify a sequence of examples over $n$ rounds. The example at each round $t$ is associated with an agent $a_{t}=\left(x_{t}, y_{t}, d_{t}\right)$ where $x_{t} \in \mathbb{R}^{d}$ is the agent's true feature vector, $y_{t} \in\{-1,1\}$ is the true label and $d_{t}: \mathbb{R}^{d} \times \mathbb{R}^{d} \rightarrow \mathbb{R}$ is a distance function that maps pairs of feature vectors to costs. We will think of $d_{t}\left(x_{t}, \hat{x}_{t}\right)$ as the cost for the agent to change his feature vector $x_{t}$ to the feature vector $\hat{x}_{t}$. If the example is positive $\left(y_{t}=1\right)$, then we say the agent is non-strategic, and if the example is negative $\left(y_{t}=-1\right)$, then we say the agent is strategic.

Each agent $a_{t}$ has a utility function $u_{t}\left(x_{t}, \beta_{t}\right)=\left\langle\beta_{t}, x_{t}\right\rangle-d_{t}\left(\hat{x}_{t}, x_{t}\right)$. In each round $t$, the interaction between the learner and the agent is the following:

(1) The learner commits to a linear classifier parameterized by $\beta_{t} \in K$, where $K$ is the set of feasible parameters.

(2) An adversary, oblivious to the learner's choices up to round $t$, selects an agent $a_{t}=\left(x_{t}, y_{t}, d_{t}\right)$.

(3) The agent $a_{t}$ sends the data point $\hat{x}_{t}$ to the learner:

- If the agent is strategic $\left(y_{t}=-1\right)$, then the agent sends the learner his best response to $\beta_{t}$ (ties broken arbitrarily):

$$
\hat{x}_{t}\left(\beta_{t}\right) \in \arg \max _{x} u_{t}(x, \beta)
$$

- If the agent is non-strategic $\left(y_{t}=1\right)$, then the agent does not modify the feature vector, and so sends $\hat{x}_{t}\left(\beta_{t}\right)=x_{t}$ to the learner.

(4) The learner observes $y_{t}$, and experiences classification loss $c_{t}\left(\beta_{t}\right)=c\left(\hat{x}_{t}, y_{t}, \beta_{t}\right)$.

We focus on two standard classification loss functions $c$. The first is logistic loss, which corresponds to logistic regression:

$$
c_{l o g}\left(\hat{x}_{t}, y_{t}, \beta_{t}\right)=\log \left(1+e^{-y_{t} \cdot\left\langle\hat{x}_{t}, \beta_{t}\right\rangle}\right)
$$


The second is hinge loss, which corresponds to a support vector machine:

$$
c_{h}\left(\hat{x}_{t}, y_{t}, \beta_{t}\right)=\max \left(0,1-y_{t} \cdot\left\langle\hat{x}_{t}, \beta_{t}\right\rangle\right)
$$

The interaction between the learner and the agent in each strategic round can be viewed as a Stackelberg game, in which the learner as the leader plays her strategy first, and then the agent as the follower best-responds. With this observation, we define the Stackelberg regret to measure the performance of the learner. ${ }^{2}$ In words, Stackelberg regret is the difference between the cumulative loss of the learner and the cumulative loss she would have experienced if she had deployed the single best classifier in hindsight $\beta^{*}$, for the same sequence of agents (who may have responded differently). More formally, for a history of play involving the $T$ agents $A=\left(a_{1}, a_{2}, \ldots, a_{T}\right)$ and the sequence of $T$ classifiers $B=\left(\beta_{1}, \beta_{2}, \ldots, \beta_{T}\right)$ the Stackelberg regret is defined as

$$
\mathcal{R}_{S}(A, B)=\sum_{t=1}^{T} c\left(\hat{x}_{t}\left(\beta_{t}\right), y_{t}, \beta_{t}\right)-\min _{\beta \in K} \sum_{t=1}^{T} c\left(\hat{x}_{t}(\beta), y_{t}, \beta\right)
$$

Observe that the regret-minimizing classifier $\beta^{*}$ is a Stackelberg equilibrium strategy for the learner in the one-shot game in which the learner first commits to a classifier $\beta^{*}$, then all the agents $\left(a_{1}, \ldots, a_{T}\right)$ simultaneously respond with $\hat{x}_{t}\left(\beta^{*}\right)$, which results in the learner experiencing classification loss $\sum_{i=1}^{n} c\left(\hat{x}_{i}\left(\beta^{*}\right), y_{i}, \beta^{*}\right)$.

In order to derive efficient algorithms with sub-linear Stackelberg regret, we will impose some restrictions the agents' distance functions $d_{t}$ (formally described in Section 3), and we will assume the Euclidean norms of the original feature vectors $x_{t}$ are bounded by a constant $R_{1}$. We also assume our feasible set of classifers $K \subseteq \mathbb{R}^{d}$ is convex, contains the unit $\ell_{2}$-ball, and has Euclidean norm bounded by $R_{2}$, such that for any $\beta \in K,\|\beta\|_{2} \leq R_{2}$.

\section{CONDITIONS FOR A CONVEX LEARNING PROBLEM}

In this section, we derive general conditions under which the learner's problem can be cast as a convex minimization problem. In each round, the learner proposes some hypothesis $\beta$ and experiences a loss that depends on the agent's best-response to $\beta$. Even when the original loss function is a convex function of $\beta$ alone, holding $\hat{x}$ and $y$ fixed, it may no longer be convex when $\hat{x}$ is chosen strategically as a function of $\beta$.

Since the learner's objective is a summation of the loss over all rounds, and the sums of convex loss functions are convex, it suffices to show convexity for the loss experienced at each fixed round $t$. We omit the subscript $t$ for cleanliness of notation.

Recall that each strategic agent $a=(x, y, d)$ (with $y=-1$ ), when facing a classifier $\beta$, will misreport his feature vector $x$ as $\hat{x}(\beta)$ by solving a maximization problem:

$$
\hat{x}(\beta) \in \underset{\hat{x} \in \mathbb{R}^{d}}{\arg \max } u(\hat{x}, x, \beta)=\underset{\hat{x} \in \mathbb{R}^{d}}{\arg \max }(\langle\hat{x}, \beta\rangle-d(\hat{x}, x))
$$

where $d(\cdot, \cdot)$ is a "distance" function modeling the cost of deviating from the original feature vector $x$. We first show that both the logistic and hinge loss objective are convex if $\langle\hat{x}(\beta), \beta\rangle$ is convex in $\beta$, and then prove the convexity of $\langle\hat{x}(\beta), \beta\rangle$ when the distance has the form $d\left(x, x^{\prime}\right)=f\left(x-x^{\prime}\right)$, for $f$ satisfying reasonable assumptions (stated in Theorem 3.3). In Section 3.2, we present a large class of distance functions which satisfy our assumptions as well as examples of seemingly natural choices which either fail to satisfy the assumptions or lead to agents not having a well-defined optimization problem, demonstrating the necessity of our restrictions on $d$.

\footnotetext{
${ }^{2}$ The same regret notion has also appeared in the context of repeated security games [2].
} 
As an abstraction, let $h(z)$ denote the learner's loss function where $z$ corresponds to $y\langle\hat{x}(\beta), \beta\rangle$. For example, if the learner is performing logistic regression, then $h(z)=\log \left(1+e^{-z}\right)$; and if the learner is building a support vector machine, then $h(z)=(1-z)_{+}$, where $(x)_{+}$denotes $\max (x, 0)$. Observe, critically, that both of these functions are non-decreasing and convex functions in $z$.

First, consider the learner's objective. She wants to minimize

$$
c(x, y, \beta)=h(y\langle\hat{x}(\beta), \beta\rangle)=h(y\langle\hat{x}(\beta, x, y), \beta\rangle) .
$$

where

$$
\hat{x}(\beta, x, y)= \begin{cases}x, & y=1, \\ \arg \max u(\hat{x}, x, \beta), & y=-1 .\end{cases}
$$

Note that we are now writing $\hat{x}(\beta, x, y)$ rather than $\hat{x}(\beta)$ to make explicit the dependence on $x$ and $y$. Where $x$ and $y$ are clear from context and fixed, we continue to write $\hat{x}(\beta)$. We note that in general, $\hat{x}(\beta, x, y)$ is not necessarily a well-defined map, as when the arg max is not unique, there may be multiple choice of $\hat{x}$ which are best-responses to the learner's choice of $\beta$, or the agent's best-response may not even be finite. However, we show in Theorem 3.3 that as long as the agent's utility function obeys some very general properties, neither of these problems occur and both the agent's and learner's optimizations are well-defined.

To begin, we make the following observation from convex analysis:

Lemma 3.1 (E.G. [19], Theorem 5.1). Let $g: \mathbb{R} \rightarrow \mathbb{R}$ be a non-increasing convex function of a single variable and $F: \mathbb{R}^{d} \rightarrow \mathbb{R}$ be convex. Then $g(-F(x))$ is convex in $x$.

This implies the following:

Theorem 3.2. Suppose, for all $(x, y)$, a strategic agent's best-response function $\hat{x}(\beta, x, y)$ satisfies the condition that the function $\beta \mapsto\langle\hat{x}(\beta), \beta\rangle$ is convex. Then logistic loss and hinge loss are convex functions of $\beta$, i.e. for all $(x, y)$, both of the following are convex:

- $(\operatorname{logistic}) c(x, y, \beta)=\log \left(1+e^{-y\langle\hat{x}(\beta), \beta\rangle}\right)$;

- (hinge) $c(x, y, \beta)=(1-y\langle\hat{x}(\beta), \beta\rangle)_{+}$.

Proof. For strategic agents, i.e. when $y=-1$, this follows immediately from Lemma 3.1 and the fact that both loss functions are non-increasing convex functions of the variable $z=\langle\hat{x}(\beta), \beta\rangle$. When $y=1$, i.e. the agent is not strategic, $c(x, y, \beta)=h(\langle\beta, x\rangle)=h(-\langle\beta,-x\rangle)$. Note that $\langle\beta,-x\rangle$ is linear (and hence convex), so the lemma applies here as well.

Therefore, in order to show the convexity of the learner's loss function $c(x, y, \beta)$ for every $x, y$, it suffices to show that for any fixed $x$ and $y,\langle\hat{x}(\beta, x, y), \beta\rangle$ is a convex function in $\beta$. In the next section, we give sufficient conditions for this to be the case.

\subsection{Sufficient Conditions}

Our main result of this section is the following theorem, which describes a set of sufficient conditions on an agent's utility function for his optimization problem to be well-defined and convex.

Theorem 3.3. Let $u(\hat{x}, x, \beta)=\langle\hat{x}, \beta\rangle-d(\hat{x}, x)$ be the strategic agent's utility function. If $d$ has the form $d(\hat{x}, x)=f(\hat{x}-x)$ where $f: \mathbb{R}^{d} \rightarrow \mathbb{R}$ satisfies:

- $f(x)>0$ for all $x \neq 0$;

- $f$ is convex over $\mathbb{R}^{d}$;

- $f$ is positive homogeneous ${ }^{3}$ of degree $r>1$.

\footnotetext{
$\overline{{ }^{3} \mathrm{~A} \text { function } f}: \mathbb{R}^{d} \rightarrow \mathbb{R}$ is positive homogeneous of degree $k$ if for any scalar $\alpha \in \mathbb{R}$ with $\alpha>0$ and vector $x \in \mathbb{R}^{d}$, we have $f(\alpha x)=\alpha^{k} f(x)$, where $k>0$. Note that $k$ need not be an integer.
} 
then the function $\beta \mapsto\langle\beta, \underset{\hat{x}}{\arg \max } u(\hat{x}, x, \beta)\rangle$ is well-defined (i.e. finite and independent of the choices of maximizer) and convex.

When these conditions hold, we can speak of the function $\beta \mapsto\langle\hat{x}(\beta), \beta\rangle$ without ambiguity even when there are multiple best responses $\hat{x}(\beta)$.

Proof. We present the proof in a series of steps, which we first outline here. The remainder of this subsection contains the formal statement and proof of each of these steps.

(1) First, we show (Corollary 3.6) that any best-response $\hat{x}(\beta)$ satisfies $\hat{x}(\beta)=x+v$, where $v$ is some subgradient of $f^{*}$ at $\beta$ and $f^{*}$ is the convex conjugate of $f$.

(2) Next, we show (Claim 2) that $f^{*}(\beta)$ is finite for all $\beta$.

(3) Then, we prove (Claim 3) that homogeneity of $f^{*}$ follows from the homogeneity of $f$.

(4) Finally, we apply a slight generalization of Euler's homogeneous function theorem, which says that if $f^{*}$ is homogenous and convex, there is a $k$ such that for any choice of subgradient $v \in \partial f^{*}(\beta)$ (the set of subgradients at $\beta$ ), we have $\langle v, \beta\rangle=k f^{*}(\beta)$. This, together with Claims 2 and 3 , implies that $\langle\hat{x}(\beta), \beta\rangle=\langle x, \beta\rangle+k f^{*}(\beta)$ for any choice of best response $\hat{x}(\beta)$, and this function is well-defined and convex.

We begin with the first step. First, we rewrite the utility function:

$$
u(\hat{x}, x, \beta)=\langle\hat{x}, \beta\rangle-f(\hat{x}-x) .
$$

We perform a change of variable from $\hat{x}$ to $w=\hat{x}-x$ and write:

$$
v(w, \beta)=u(\hat{x}, x, \beta)=\langle x, \beta\rangle+\langle w, \beta\rangle-f(w) .
$$

The first step only relies on the convexity of $f$. Let

$$
w^{*}(\beta)=\underset{w \in \mathbb{R}^{d}}{\arg \max } v(w, \beta)
$$

be the set of maximizers of $v$ given $\beta$. We note that $w^{*}(\beta)$ is a convex set since $v(w, \beta)$ is concave in $w$ for any $\beta$. Note that if $f$ is differentiable, $w^{*}(\beta)$ is a singleton set.

Definition 3.4. The convex conjugate of a function $f: \mathbb{R}^{d} \rightarrow \mathbb{R}$, denoted $f^{*}: \mathbb{R}^{d} \rightarrow \mathbb{R}$ is the function defined as

$$
f^{*}\left(x^{*}\right)=\sup _{x \in \mathbb{R}^{d}}\left(\left\langle x^{*}, x\right\rangle-f(x)\right) .
$$

$f^{*}$ is always convex (even when $f$ is not) but is not necessarily finite. However, our positivity and homogeneity assumptions imply that it must be finite everywhere, as we will prove in Claim 2.

CLAim 1. The set $w^{*}(\beta)$ is equal to $\partial f^{*}(\beta)$, the set of subgradients of $f^{*}$ at $\beta$.

Proof. Fix a $\beta$. Recall that

$$
v(w, \beta)=\langle x, \beta\rangle+\langle w, \beta\rangle-f(w) .
$$

We can ignore $\langle x, \beta\rangle$ as it is a constant with respect to a fixed $\beta$. We have that

$$
\begin{aligned}
w \in w^{*}(\beta) & \Leftrightarrow 0 \in \partial[\langle w, \beta\rangle-f(w)] \\
& \Leftrightarrow \beta \in \partial f(w) \\
& \Leftrightarrow w \in \partial f^{*}(\beta) .
\end{aligned}
$$

The first equivalence follows from the optimality of $w$. The second follows from computation. To show the last equivalence, we apply the following theorem from convex analysis: 
Theorem 3.5 (Rockafellar [19], Theorem 23.5; cF Corollary 10.1.1, Theorem 7.1). Let $f$ : $\mathbb{R}^{d} \rightarrow \mathbb{R}$ be convex and finite everywhere. Then

$$
x^{*} \in \partial f(x) \Leftrightarrow x \in \partial f^{*}\left(x^{*}\right) .
$$

Therefore, we have

COROLlary 3.6. The Strategic agent's best-response set is

$$
\underset{\hat{x} \in \mathbb{R}^{d}}{\arg \max } u(\hat{x}, x, \beta)=x+w^{*}(\beta)=x+\partial f^{*}(\beta),
$$

which concludes the first step of the proof of Theorem 3.3.

We now move on to the second step. We want to show that $f^{*}$ is always finite and that the set of best responses of the strategic agent is non-empty and bounded.

CLAIM 2. Under the assumptions in Theorem 3.3, $f^{*}(\beta)$ is finite for all $\beta \in \mathbb{R}^{d}$.

See the full version for the proof.

We now turn our attention to the set of best responses. The following theorem guarantees that the strategic agents' best response correspondence is well behaved:

Theorem 3.7 (Rockafellar [19], Theorem 23.4). Suppose $g: \mathbb{R}^{d} \rightarrow \mathbb{R}$ is convex and finite everywhere. Then $\partial g(x)$ is a non-empty and compact subset of $\mathbb{R}^{d}$ for all $x \in \mathbb{R}^{d}$.

Since we showed that $f^{*}$ is convex and finite everywhere, this, together with Corollary 3.6, implies:

Corollary 3.8. Under the assumption of Theorem 3.3, the set $\arg \max u(\hat{x}, x, \beta)$ is non-empty and bounded for all $\beta \in \mathbb{R}^{d}$. $\hat{x} \in \mathbb{R}^{d}$

The boundedness of the set $\arg \max u(\hat{x}, x, \beta)$ is not directly used in the proof of Theorem 3.3, but $\hat{x} \in \mathbb{R}^{d}$

it excludes the unrealistic situation in which the strategic agent's best response can be arbitrarily far from the origin. Thus we have completed the second step of the proof of Theorem 3.3.

The third step consists of showing the following claim:

Claim 3. If $f: \mathbb{R}^{d} \rightarrow \mathbb{R}$ is convex and homogeneous of degree $r>1$, then $f^{*}: \mathbb{R}^{d} \rightarrow \mathbb{R}$ is convex and homogeneous of degree $s>1$, where $\frac{1}{r}+\frac{1}{s}=1$.

Throughout the rest of the paper, $(r, s)$ (and also $(p, q)$ which we will use when discussing norms) will refer to such a pair of numbers unless stated otherwise. For the proof of Claim 3, see the full version.

Finally we move to the fourth step of the proof. First we use a slightly more general version of Euler's theorem on homogeneous functions.

Theorem 3.9 (Euler). Assume $g$ is convex and homogeneous of degree $k>0$, then for any $v \in \partial g(x)$, $\langle v, x\rangle=k g(x)$.

We make two quick observations about this version of Euler's theorem. First, this is a slightly more general statement than the standard statement of Euler's theorem in that it can deal with non-differentiable functions. Secondly, the value of the inner product is independent of the choice of the subgradient, which is interesting. Curious readers may refer to the full version ${ }^{4}$ for the proof.

\footnotetext{
${ }^{4}$ Available on ArXiv at https://arxiv.org/abs/1710.07887.
} 
Combining Claim 3 and Euler's theorem, if $f$ is convex and homogeneous of degree $r>1$, we have that

$$
\langle\hat{x}(\beta), \beta\rangle=\langle x, \beta\rangle+s f^{*}(\beta)
$$

for any $\hat{x}(\beta) \in \arg \max u(\hat{x}, x, \beta)$. This shows that $\langle\hat{x}(\beta), \beta\rangle$ is a well-defined convex function of $\beta$. $\hat{x} \in \mathbb{R}^{d}$

This concludes the proof of Theorem 3.3.

\subsection{Example distance functions}

In this section we provide a large class of functions $f$ that satisfy the assumptions in Theorem 3.3. (Recall that we focus on distance functions of the form $d\left(x, x^{\prime}\right)=f\left(x-x^{\prime}\right)$.) We will also see that for this class of functions, we can derive simple closed form expressions for $f^{*}$, which will help us determine the convergence rate of the optimization procedure we develop in Section 4. We will also see some examples that violate our assumptions, which demonstrate the necessity of those assumptions; without them, agent best responses are not necessarily well-defined.

CLAIM 4. For any arbitrary norm $\|\cdot\|$ on $\mathbb{R}^{d}$ and any $r>1$

$$
f(x)=\frac{1}{r}\|x\|^{r}
$$

satisfies the conditions of Theorem 3.3. Namely,

- $f(x)>0$ for all $x \neq 0$;

- $f$ is convex over $\mathbb{R}^{d}$;

- $f$ is positive homogeneous of degree $r>1$.

Furthermore, let $\|\cdot\|_{*}$ be the dual norm of $\|\cdot\| \cdot f^{*}$ has the following form:

$$
f^{*}(\beta)=\frac{1}{s}\|\beta\|_{*}^{s} .
$$

Here $1 / r+1 / s=1$.

The conditions can be easily checked. See the full version for the derivation of $f^{*}$.

CoRollary 3.10. For any norm $\|\cdot\|$ on $\mathbb{R}^{d}$, any $r>1$ and any invertible linear transformation $A: \mathbb{R}^{d} \rightarrow \mathbb{R}^{d}$, the function

$$
f(x)=\frac{1}{r}\|A x\|^{r}
$$

satisfies all the conditions in Theorem 3.3. In this case, $f^{*}$ has the following form:

$$
f^{*}(\beta)=\frac{1}{s}\left\|\left(A^{T}\right)^{-1} \beta\right\|_{*}^{s} .
$$

Proof. Since $\|A(\cdot)\|$ is also a norm, the conditions follow from Claim 4 . The expression for $f^{*}$ follows from the identity

$$
\langle x, \beta\rangle-g(A x)=\left\langle A x,\left(A^{T}\right)^{-1} \beta\right\rangle-g(A x)
$$

In particular, if $\|\cdot\|_{2}$ is the Euclidean 2-norm and $r=s=2, f(x)=\frac{1}{2}\|A x\|^{2}$ can be viewed as a form of Mahalanobis distance, commonly studied in the metric learning literature [17].

Combining Corollary 3.10, Theorem 3.2, and Theorem 3.3, we can naturally extend this general form of distance functions. 
Corollary 3.11. For any norm $\|\cdot\|$ on $\mathbb{R}^{d}$, any invertible linear transformation $A: \mathbb{R}^{d} \rightarrow \mathbb{R}^{d}$, and any $r>1$, if an agent's utility is of the form

$$
u(\hat{x}, x, \beta)=\langle\hat{x}, \beta\rangle-\frac{1}{r}\|A x-A \hat{x}\|^{r},
$$

then both the logistic loss and and hinge loss are convex functions of $\beta$, assuming the agent best-responds to $\beta$.

Norms and Non-examples. We present two non-examples of distance functions, which illustrate the following points:

(1) There exist functions $f$ which violate the condition that $f$ is nonvanishing except at the origin, for which strategic agents might not have finite best responses, and;

(2) When we let the power $r=1$ (violating the $r>1$ condition), again, strategic agents might not have finite best-responses.

Our first example shows that the linear transformation $A$ we used in Corollary 3.11 must be invertible, i.e. full rank. The second example shows that $f(x)=\|x\|$ where $\|\cdot\|$ is a norm (not raised to any power $r>1$ ) also fails to constrain the agent's best-responses to be finite.

To illustrate the first point, consider a utility function of the form used in Corollary 3.11 but with $A$ having non-trivial kernel. Assume $v \in \operatorname{ker} A, v \neq 0$. Note that $f(v)=0$, violating our non-degeneracy condition. When $\hat{x}=x+\lambda v$, for any $\lambda \in \mathbb{R}$, the deviation incurs no cost since $v$ is in the kernel of $A$. Whenever $\beta$ is not orthogonal to $v$, therefore, the agents have no finite best response; they can increase their utility to $+\infty$ by letting $\lambda$ go to $+\infty$ or $-\infty$. Hence, for this choice of $f$, best-responses for the agents are not even well-defined.

It is also natural to ask what happens when $f$ is a norm, rather than a norm raised to some power $r$ greater than one. After all, any norm is positive homogeneous of degree one. In this case too, agent best responses are not necessarily well defined. The issue is that norms do not exhibit diminishing returns to the agent as he moves $\hat{x}$ increasingly far from $x$. Thus, the agent's best response would always be either to not manipulate at all (resulting in truthfully setting $\hat{x}=x$ ), or else to manipulate his data arbitrarily far from $x$, with arbitrarily high reward achievable. Formally,

Claim 5. Suppose the agent's distance function is $d(\hat{x}, x)=\|\hat{x}-x\|$ for some norm $\|\cdot\|$, and let $\|\cdot\|_{*}$ be the dual norm. Then his best response is $\hat{x}=x$ for $\|\beta\|_{*} \leq 1$; and if $\|\beta\|_{*}>1$, his best response is undefined with arbitrarily high utility achievable.

Proof. Let $f$ be some norm, i.e. $f(z)=\|z\|$. The agent wishes to solve

$$
\begin{aligned}
\sup _{\hat{x}}(\langle\hat{x}, \beta\rangle & -f(\hat{x}-x))= \\
& =\langle x, \beta\rangle+\sup _{\hat{x}}(\langle\hat{x}-x, \beta\rangle-f(\hat{x}-x)) \\
& =\langle x, \beta\rangle+\sup _{x^{\prime}}\left\langle x^{\prime}, \beta\right\rangle-f\left(x^{\prime}\right) \\
& =\langle x, \beta\rangle+f^{*}(\beta)
\end{aligned}
$$

where $f^{*}$, the conjugate of $f$, is the $(0-\infty)$ indicator function of the unit ball in the dual norm [19], namely

$$
f^{*}(\beta)= \begin{cases}0 & \|\beta\|_{*} \leqslant 1 \\ \infty & \text { otherwise }\end{cases}
$$

This implies the claim. 


\section{REGRET MINIMIZATION}

In this section, we will present an online convex optimization method that has sub-linear Stackelberg regret. Our algorithm is a slight variant of the bandit algorithm in [12], which only uses zeroth-order feedback. That is, it only observes information of the form (input, function value). In our setting, the learner always has access to zeroth-order information, as she observes the loss function at a particular choice of parameters $\beta_{t}$. However, when a non-strategic agent arrives, the learner can also deduce first-order information, that is, the subgradient of the loss evaluated at $\beta_{t}$. Our algorithm takes advantage of this mixture of feedback and performs a full subgradient descent update for every non-strategic round. As a result, we obtain a regret rate that depends on the fraction of strategic agents that show up.

\subsection{Convex Optimization with Mixture Feedback}

In this section, we abstract away from the particular forms of the loss function until they become relevant. At a high level, our algorithm is running (stochastic) subgradient descent to minimize a sequence of convex loss functions $c_{t}(\beta)$ while the learner proposes a sequence of parameters $\beta_{t}$. The key challenge is to obtain subgradient feedback based on the agent's data $(\hat{x}, y)$. In each non-strategic round, we can explicitly compute a subgradient in $\partial c_{t}\left(\beta_{t}\right)$. In other words, we have first-order information. In each strategic round, however, since $\hat{x}_{t}$ is a function of $\beta_{t}$, we do not have direct access to the subgradients of $c_{t}$, and only see the "zeroth-order" information $c_{t}\left(\beta_{t}\right)$.

To estimate a subgradient with this information, we utilize the approach of Flaxman et al. [12] and minimize a "smoothed" version of the classification loss. In particular, for any $c_{t}$ and parameter $\delta$, the $\delta$-smoothed version of $c_{t}$ is defined as:

$$
\tilde{c_{t}}(\beta)=\mathbb{E}\left[c_{t}(\beta+\delta S)\right]
$$

where $S$ is a random vector drawn from the uniform distribution over the unit sphere in $\mathbb{R}^{d}$. Notice that $\tilde{c}_{t}$ is both convex and differentiable. Flaxman et al. [12] provides a method to obtain an unbiased estimate for the gradient of the smoothed loss $\tilde{c}$. This is done by perturbing $\beta_{t}$ to get a noisy $\beta_{t}^{+}$ and then evaluating the classification loss at $\beta_{t}^{+}$. More formally:

LEMMA 4.1 ([12]). Let $\beta_{t}^{+}=\beta_{t}+\delta \cdot S$ and $G_{t}=\frac{d}{\delta} c_{t}\left(\beta_{t}^{+}\right) S$, where $S$ is a random vector drawn from the uniform distribution over the unit sphere in $\mathbb{R}^{d}$. Then $\mathbb{E}\left[G_{t}\right]=\nabla \tilde{c_{t}}\left(\beta_{t}\right)$.

Note that the perturbed point $\beta_{t}^{+}$may fall outside of the feasible set $K$. To prevent that, we will optimize over a strict subset $K_{\delta}=\{(1-\delta) \beta \mid \beta \in K\}$. Then we can guarantee that for any $\beta \in K_{\delta}$, the $\delta$-ball centered at $\beta$ is fully contained in $K .^{5}$

Let $\theta=\frac{\#\left\{t: y_{t}=-1\right\}}{n}$ be the fraction of rounds in which the learner interacts with a strategic agent. For simplicity, we assume for now that $\theta$ is known to the algorithm in advance, and use it to set the parameters $\eta$ and $\delta$ in our algorithm. However, as we discuss at the end of this section, any upper bound $\hat{\theta}$ on $\theta$ suffices, and can be used in its place, at the cost of only an additional additive constant to the final regret bound. We are now ready to present our main algorithm in Algorithm 1.

Now we will proceed to bound the regret rate of our algorithm. To facilitate our analysis, we will make the following well-behaved assumption on the loss function $c_{t}$ for all rounds $t$ :

- $\left|c_{t}(\beta)\right| \leqslant M$ for any $\beta \in K$, and

- $c_{t}$ is $L$-Lipschitz (with respect to the $\ell_{2}$ norm) over the set $K$.

In the full version, we work out the parameters $M$ and $L$ for both logistic loss and hinge loss, along with distance function of the form $d_{t}(x, y)=f\left(A_{t}(x-y)\right)$ for the strategic agents.

\footnotetext{
${ }^{5}$ Any $\beta_{t}^{+}=(1-\delta) \beta+\delta v$ for some $\beta \in K$ and some unit vector $v$. We assume $K$ contains the unit ball, in particular, both $v$ and $\beta$ are $K$, and $\beta_{t}^{+}$is a convex combination of them, so it also lies in $K$.
} 
ALGORITHM 1: Convex optimization with mixture feedback

Input: smoothing parameter $\delta=\theta^{1 / 4} \cdot \sqrt{\frac{d M R}{L(R+3)}} \cdot n^{-1 / 4}$, subset to optimize over $K_{\delta} \subset K$, step size $\eta=R / \sqrt{n\left(\theta \cdot \frac{d^{2} M^{2}}{\delta^{2}}+(1-\theta) L^{2}\right)}$

For each round $t$ :

Let $S_{t}$ be drawn uniformly from the unit sphere;

Let $\beta_{t}^{+}=\beta_{t}+\delta \cdot S_{t}$;

Query at $\beta_{t}^{+}$, observe corresponding $\hat{x}_{t}, y_{t}$, and suffer loss $c_{t}\left(\beta_{t}^{+}\right)$;

Compute stochastic subgradient:

If $y_{t}=1$, then set $g_{t}$ as a subgradient in $\partial c_{t}\left(\beta_{t}\right)$; otherwise set $g_{t}=\frac{d}{\delta} \cdot c_{t}\left(\beta_{t}^{+}\right) \cdot S_{t}$.

Update: $\beta_{t+1}=\Pi_{K_{\delta}}\left(\beta_{t}-\eta g_{t}\right)$, where $\Pi_{K_{\delta}}$ is the Euclidean projection map onto the set $K_{\delta}$.

The following lemma allows us to bound the regret separately in non-strategic and strategic rounds, and also bounds the additional regret due to the $\delta$-smoothing of our losses.

LemMA 4.2. Let $T_{1}:=\left\{t: y_{t}=1, t=1,2, \ldots, n\right\}$ be the non-strategic rounds and $T_{-1}:=\left\{t: y_{t}=\right.$ $-1, t=1,2, \ldots, n\}$ be the strategic rounds. Then for any $\beta \in K$,

$$
\mathbb{E}\left[\sum_{t=1}^{n} c_{t}\left(\beta_{t}^{+}\right)-c_{t}(\beta)\right] \leqslant \mathbb{E}\left[\sum_{t \in T_{-1}} \tilde{c_{t}}\left(\beta_{t}\right)-\tilde{c_{t}}(\beta)\right]+\mathbb{E}\left[\sum_{t \in T_{1}} c_{t}\left(\beta_{t}\right)-c_{t}(\beta)\right]+3 n L \delta,
$$

where the expectation is taken over the randomness of the algorithm.

Proof. Fix any $\beta \in K_{\delta}$. Since the vector $S$ is drawn from the unit sphere, $\left|c_{t}(\beta+\delta S)-c_{t}(\beta)\right| \leqslant$ $L\|\delta S\|=L \delta$. Hence by taking expectation over $S$,

$$
\left|\tilde{c_{t}}(\beta)-c_{t}(\beta)\right| \leqslant L \delta .
$$

Recall that $\beta_{t}^{+}=\beta_{t}+\delta \cdot S$, by Lipschitz property again, $\left|c_{t}\left(\beta_{t}^{+}\right)-c_{t}\left(\beta_{t}\right)\right| \leqslant L \delta$. For any $t \in T_{-1}$, we have

$$
\begin{aligned}
\left|c_{t}\left(\beta_{t}^{+}\right)-\tilde{c_{t}}\left(\beta_{t}\right)\right| & \leqslant\left|c_{t}\left(\beta_{t}^{+}\right)-c_{t}\left(\beta_{t}\right)\right|+\left|c_{t}\left(\beta_{t}\right)-\tilde{c_{t}}\left(\beta_{t}\right)\right| \\
& \leqslant L \delta+L \delta=2 L \delta .
\end{aligned}
$$

Now, we want to bound the difference between the two expressions, $c_{t}\left(\beta_{t}^{+}\right)-c_{t}(\beta)$ and $\tilde{c_{t}}\left(\beta_{t}\right)-\tilde{c_{t}}(\beta)$. By Equation (3), we have $\left|c_{t}\left(\beta_{t}^{+}\right)-\tilde{c_{t}}\left(\beta_{t}\right)\right| \leq 2 L \delta$, and by Equation (2), we have $\left|c_{t}(\beta)-\tilde{c_{t}}(\beta)\right| \leq L \delta$. It follows that

$$
c_{t}\left(\beta_{t}^{+}\right)-c_{t}(\beta) \leqslant \tilde{c_{t}}\left(\beta_{t}\right)-\tilde{c_{t}}(\beta)+3 L \delta
$$

Similarly, for any $t \in T_{1}$, we can bound

$$
c_{t}\left(\beta_{t}^{+}\right)-c_{t}(\beta) \leqslant c_{t}\left(\beta_{t}\right)-c_{t}(\beta)+L \delta .
$$

Summing over all rounds $t$, we recover the stated bound.

By bounding the regret separately in non-strategic and strategic rounds, we can then bound the Stackelberg regret of Algorithm 1.

TheOrem 4.3. For any sequence of agents $A=\left(a_{1}, \ldots, a_{n}\right)$, Algorithm 1 will output a sequence of classifiers $B=\left(\beta_{1}, \ldots, \beta_{n}\right)$ such that the expected Stackelberg regret satisfies

$$
\mathbb{E}\left[\mathcal{R}_{S}(A, B)\right] \leqslant \frac{\eta}{2}\left[n \theta \cdot \frac{d^{2} M^{2}}{\delta^{2}}+n(1-\theta) L^{2}\right]+\frac{1}{2 \eta} R^{2}+3 n L \delta+n L R \delta .
$$


Proof. Our proof essentially follows from the standard analysis for online subgradient descent. We will first bound

$$
\mathbb{E}\left[\sum_{t \in T_{-1}} \tilde{c_{t}}\left(\beta_{t}\right)-\tilde{c_{t}}\left(\beta^{*}\right)\right]+\mathbb{E}\left[\sum_{t \in T_{1}} c_{t}\left(\beta_{t}\right)-c_{t}\left(\beta^{*}\right)\right]
$$

which then allows us to apply Lemma 4.2. By convexity, we get for any $\beta \in K$,

$$
\tilde{c_{t}}\left(\beta_{t}\right)-\tilde{c_{t}}(\beta) \leqslant\left\langle\nabla \tilde{c_{t}}\left(\beta_{t}\right), \beta_{t}-\beta\right\rangle
$$

and for any subgradient $G_{t}(\beta) \in \partial c_{t}\left(\beta_{t}\right)$,

$$
\begin{gathered}
c_{t}\left(\beta_{t}\right)-c_{t}(\beta) \leqslant\left\langle G_{t}(\beta), \beta_{t}-\beta\right\rangle . \\
\tilde{c_{t}}\left(\beta_{t}^{+}\right)-\tilde{c_{t}}(\beta)=\left[\tilde{c_{t}}\left(\beta_{t}^{+}\right)-\tilde{c_{t}}\left(\beta_{t}\right)\right]+\left[\tilde{c_{t}}\left(\beta_{t}\right)-\tilde{c_{t}}(\beta)\right] \\
\leqslant L_{0} \delta+\tilde{c_{t}}\left(\beta_{t}\right)-\tilde{c_{t}}(\beta)
\end{gathered}
$$

Recall that for any $t \in T_{1}, g_{t} \in \partial c_{t}\left(\beta_{t}\right)$, and by Lemma 4.1, we know for $t \in T_{-1}$, we can write $g_{t}=\nabla \tilde{c_{t}}\left(\beta_{t}\right)-\xi_{t}$ where $\xi_{t}$ is a random vector with zero mean. Let $\beta^{\prime} \in \arg \min _{\beta \in K_{\delta}} \sum_{t=1}^{n} c_{t}(\beta)$, then

$$
\sum_{t \in T_{-1}}\left[\tilde{c_{t}}\left(\beta_{t}\right)-\tilde{c_{t}}\left(\beta^{\prime}\right)\right]+\sum_{t \in T_{1}}\left[c_{t}\left(\beta_{t}\right)-c_{t}\left(\beta^{\prime}\right)\right] \leqslant \sum_{t=1}^{n}\left\langle g_{t}, \beta_{t}-\beta^{\prime}\right\rangle+\sum_{t \in T_{-1}}\left\langle\xi_{t}, \beta_{t}-\beta^{\prime}\right\rangle .
$$

Taking the expectation, we have

$$
\mathbb{E}\left[\sum_{t \in T_{-1}} \tilde{c_{t}}\left(\beta_{t}\right)-\tilde{c_{t}}\left(\beta^{\prime}\right)\right]+\mathbb{E}\left[\sum_{t \in T_{1}} c_{t}\left(\beta_{t}\right)-c_{t}\left(\beta^{\prime}\right)\right] \leqslant \mathbb{E} \sum_{t=1}^{n}\left\langle g_{t}, \beta_{t}-\beta^{\prime}\right\rangle .
$$

Now we can employ the standard cosine law trick (see e.g. [9], [5]) and get:

$$
\left\langle g_{t}, \beta_{t}-\beta^{\prime}\right\rangle \leqslant \frac{\eta}{2}\left\|g_{t}\right\|^{2}+\frac{1}{2 \eta}\left(\left\|\beta^{\prime}-\beta_{t}\right\|^{2}-\left\|\beta^{\prime}-\beta_{t+1}\right\|^{2}\right)
$$

Summing them up, telescoping and leaving out the negative term yields:

$$
\sum_{t=1}^{n}\left\langle g_{t}, \beta_{t}-\beta^{\prime}\right\rangle \leqslant \frac{\eta}{2} \sum_{t=1}^{n}\left\|g_{t}\right\|^{2}+\frac{1}{2 \eta}\left\|\beta^{\prime}-\beta_{1}\right\|^{2}
$$

Since we have

$$
\left\|g_{t}\right\| \leqslant \begin{cases}L, & y_{t}=0 \\ \frac{d}{\delta} \cdot M, & y_{t}=1,\end{cases}
$$

Combining (4) and (5) and using the above bound for $g_{t}$, we get

$$
\mathbb{E}\left[\sum_{t \in T_{-1}} \tilde{c_{t}}\left(\beta_{t}\right)-\tilde{c_{t}}\left(\beta^{\prime}\right)\right]+\mathbb{E}\left[\sum_{t \in T_{1}} c_{t}\left(\beta_{t}\right)-c_{t}\left(\beta^{\prime}\right)\right] \leqslant \frac{\eta}{2}\left[n \theta \cdot \frac{d^{2} M^{2}}{\delta^{2}}+n(1-\theta) L^{2}\right]+\frac{1}{2 \eta} R^{2} .
$$

Finally, we need bound the additional error we incur for restricting to the subset $K_{\delta}$. Since for any point $\beta \in K$, the closet point to $\beta$ in $K_{\delta}$ has $\ell_{2}$ distance no more than $\delta R$, by the Lipschitz property of each $c_{t}$, we know

$$
\sum_{t=1}^{n} c_{t}\left(\beta^{\prime}\right)-\min _{\beta \in K} \sum_{t=1}^{n} c_{t}(\beta) \leq n L R \delta
$$

Our regret bound then follows from Equation (6) and Equation (7).

In Corollary 4.4, we provide a regret rate for three different regimes of $p$ (hiding dependence on $M, L$, and $R$ ). 
CoROLlary 4.4. For different ranges of $\theta$, we can bound the expected Stackelberg regret by

$$
\mathbb{E}\left[\mathcal{R}_{S}(A, B)\right] \leqslant \begin{cases}O(\sqrt{n}), & \theta=0, \\ O\left(\sqrt{d} n^{3 / 4}\right), & \theta=\Omega(1), \\ O\left(\sqrt{d} n^{1-\frac{1+\gamma}{4}}\right) & \theta=O\left(n^{-\gamma}\right)\end{cases}
$$

Proof. Plugging the algorithm parameter $\eta=R / \sqrt{n\left(\theta \cdot \frac{d^{2} M^{2}}{\delta^{2}}+(1-\theta) L^{2}\right)}$ into the regret bound in Theorem 4.3,

$$
\mathcal{R}_{S} \leqslant \sqrt{\theta \cdot \frac{d^{2} M^{2}}{\delta^{2}}+(1-\theta) L^{2}} \cdot \sqrt{n} \cdot R+n \delta L(R+3) .
$$

Using $\sqrt{a+b} \leqslant \sqrt{a}+\sqrt{b}$, we can further simplify the bound:

$$
\mathcal{R}_{S} \leqslant \sqrt{n \theta} \cdot \frac{d M R}{\delta}+L R \sqrt{n(1-\theta)}+n \delta L(R+3) .
$$

Plugging in $\delta=\theta^{1 / 4} \cdot \sqrt{\frac{d M R}{L(R+3)}} \cdot n^{-1 / 4}$ yields:

$$
\mathcal{R}_{S} \leqslant L R \sqrt{n(1-\theta)}+n^{3 / 4} \sqrt{d M L R(R+3)} \theta^{1 / 4} .
$$

The rest of the result follows by setting $\theta=0, \Omega(1)$ or $O\left(n^{-\gamma}\right)$.

Relaxing $\theta$ to $\hat{\theta}$. Let $\hat{\theta}$ be an upper bound on the true fraction of strategic agents in rounds $1,2, \ldots, n$, i.e. $\theta \leqslant \hat{\theta}$. We briefly explain why $\hat{\theta}$ can be used in place of $\theta$ in setting the parameters of Algorithm 1, and Corollary 4.4 still holds (with $\hat{\theta}$ replacing $\theta$ in the bound.) First, consider the case in which $\frac{d M}{\delta} \geqslant L$. In this case, the right hand side of the bound in Theorem 4.3 is increasing in $\theta$, so we can replace $\theta$ with $\hat{\theta}$ and still get a correct regret bound:

$$
\mathbb{E}\left[\mathcal{R}_{S}(A, B)\right] \leqslant \frac{\eta}{2}\left[n \hat{\theta} \cdot \frac{d^{2} M^{2}}{\delta^{2}}+n(1-\hat{\theta}) L^{2}\right]+\frac{1}{2 \eta} R^{2}+3 n L \delta+n L R \delta .
$$

Thus, we can carry out the calculations from the proof of Corollary 4.4, again with $\hat{\theta}$ replacing $\theta$. Finally, it is not hard to show that when $n \geqslant\left(\frac{L}{d M}\right)^{2}$, we are in the case in which $\frac{d M}{\delta} \geqslant L$. Since we assume $c_{t}(\beta) \leqslant M$, each round incurs regret at most $2 M$. Therefore in the remaining case in which $n<\left(\frac{L}{d M}\right)^{2}$, we can upper bound the regret by $2 M \cdot n \leqslant 2 M \cdot\left(\frac{L}{d M}\right)^{2}$. Hence, we obtain the regret bound

$$
\mathcal{R}_{S} \leqslant \max \left\{L R \sqrt{n(1-\hat{\theta})}+n^{3 / 4} \sqrt{d M L R(R+3)} \hat{\theta}^{1 / 4}, \frac{2 L^{2}}{d^{2} M}\right\} .
$$

The new (second) term in the maximum is independent of $n$, and so does not change the asymptotic bound stated in Corollary 4.4.

Applying other tools for bandit convex optimization. We can in fact apply other tools for bandit convex optimization to minimize regret. For example, we can use the algorithm in [10], which will achieve a Stackelberg regret rate of $\tilde{O}\left(d^{9.5} \sqrt{n}\right)$ even when all the agents are strategic (that is $p=1$ ). Our algorithm, which is a variant of [12], has a regret rate with a worse dependence on the time horizon, but has a milder dependence on the dimension. Furthermore, it also permits a natural interpolation between the fully strategic and fully non-strategic settings (as shown in Corollary 4.4). 
Deriving the Constants. In the full version, we first illustrate how to determine the constants $L$ and $M$ that appear in our regret bound for general classes of cost functions $d(x, y)$, namely any abstract norm raised to some power greater than 1 :

$$
d\left(x, x^{\prime}\right)=\frac{1}{r}\left\|x-x^{\prime}\right\|^{r} .
$$

The constant $C$ in the inequality $\|x\|_{2} \leqslant C\|x\|$ can be used to determine $L$ and $M$. See the full version for details.

We also give explicit expressions for $L$ and $M$ when the abstract norm is some $p$-norm with $p \geqslant 1$ in the full version.

All assumptions and the resulting fully expanded regret bound are described in the full version. We state here a noteworthy special case that has an especially mild dependence on the dimension $d:$

When $1 \leqslant p \leqslant 2$, and for any power $r>1$, Algorithm 1 has regret bound:

$$
\mathbb{E}\left[\mathcal{R}_{S}(A, B)\right] \leqslant \begin{cases}O(\sqrt{n}), & \theta=0, \\ O\left(\sqrt{d} n^{3 / 4}\right), & \theta=\Omega(1), \\ O\left(\sqrt{d} n^{1-\frac{1+\gamma}{4}}\right), & \theta=O\left(n^{-\gamma}\right) .\end{cases}
$$

i.e. for $p$ norms when $1 \leqslant p \leqslant 2$, regardless of the power $r>1$, we obtain a fixed (and mild) dependence on $d$. When $p>2$, the situation changes: our dependence on $d$ grows with $p$, and shrinks with $r$.

\section{DISCUSSION AND OPEN QUESTIONS}

Our work suggests a number of interesting directions. Broadly speaking, these can be grouped into two thrusts: broadening the class of problems to which our approach can be applied, and weakening the assumptions that need to be made about the agents. Here we list a concrete example of each type of question.

The broad approach we take in this paper is to identify classes of utility functions for the strategic agents, that, when paired with natural cost functions for the learner, lead to a convex objective, even in the strategic setting. However, this precludes several natural utility functions for the agents: most notably, utility functions that are defined in terms of $0 / 1$ classification loss. What can be done in the face of non-convexity? When agent cost functions $d$ are known and separable, [14] show that even for 0/1 loss, Stackelberg equilibria have structure that allows for their efficient computation, despite non-convexity of the learner's objective. Can similar structure be taken advantage of in the setting we consider, when agent cost functions are not known to the learner?

Next, the overarching motivation of this paper is to weaken the assumptions on the knowledge of the learner necessary to solve the strategic classification problem. We should also aim to weaken other assumptions. For example, we assume in this paper that strategic agents play exact best responses - i.e. the always exactly optimize their utility function. What if strategic agents are not perfect - i.e. they are only guaranteed to play approximate best responses (or, perhaps only usually guaranteed to). Is it possible to give optimization procedures with guarantees that are robust to strategic agent imperfection?

\section{REFERENCES}

[1] Kareem Amin, Rachel Cummings, Lili Dworkin, Michael Kearns, and Aaron Roth. 2015. Online Learning and Profit Maximization from Revealed Preferences.. In AAAI. 770-776.

[2] Maria-Florina Balcan, Avrim Blum, Nika Haghtalab, and Ariel D. Procaccia. 2015. Commitment Without Regrets: Online Learning in Stackelberg Security Games. In Proceedings of the Sixteenth ACM Conference on Economics and Computation, EC '15, Portland, OR, USA, fune 15-19, 2015. 61-78. https://doi.org/10.1145/2764468.2764478 
[3] Maria-Florina Balcan, Amit Daniely, Ruta Mehta, Ruth Urner, and Vijay V Vazirani. 2014. Learning economic parameters from revealed preferences. In International Conference on Web and Internet Economics. Springer, 338-353.

[4] Eyal Beigman and Rakesh Vohra. 2006. Learning from revealed preference. In Proceedings of the 7th ACM Conference on Electronic Commerce. ACM, 36-42.

[5] Aharon Ben-Tal and Arkadi Nemirovski. 2001. Lectures on modern convex optimization: analysis, algorithms, and engineering applications. SIAM.

[6] Michael Brückner, Christian Kanzow, and Tobias Scheffer. 2012. Static prediction games for adversarial learning problems. Journal of Machine Learning Research 13, Sep (2012), 2617-2654.

[7] Michael Brückner and Tobias Scheffer. 2009. Nash equilibria of static prediction games. In Advances in neural information processing systems. 171-179.

[8] Michael Brückner and Tobias Scheffer. 2011. Stackelberg games for adversarial prediction problems. In Proceedings of the 17th ACM SIGKDD international conference on Knowledge discovery and data mining. ACM, 547-555.

[9] Sébastien Bubeck. 2015. Convex Optimization: Algorithms and Complexity. Foundations and Trends in Machine Learning 8, 3-4 (2015), 231-357. https://doi.org/10.1561/2200000050

[10] Sébastien Bubeck, Yin Tat Lee, and Ronen Eldan. 2017. Kernel-based methods for bandit convex optimization. (2017), 72-85. https://doi.org/10.1145/3055399.3055403

[11] Nilesh Dalvi, Pedro Domingos, Sumit Sanghai, and Deepak Verma. 2004. Adversarial classification. In Proceedings of the tenth ACM SIGKDD international conference on Knowledge discovery and data mining. ACM, 99-108.

[12] Abraham D Flaxman, Adam Tauman Kalai, and H Brendan McMahan. 2005. Online convex optimization in the bandit setting: gradient descent without a gradient. In Proceedings of the sixteenth annual ACM-SIAM symposium on Discrete algorithms. Society for Industrial and Applied Mathematics, 385-394.

[13] Michael Großhans, Christoph Sawade, Michael Brückner, and Tobias Scheffer. 2013. Bayesian games for adversarial regression problems. In International Conference on Machine Learning. 55-63.

[14] Moritz Hardt, Nimrod Megiddo, Christos Papadimitriou, and Mary Wootters. 2016. Strategic classification. In Proceedings of the 2016 ACM Conference on Innovations in Theoretical Computer Science. ACM, 111-122.

[15] Shahin Jabbari, Ryan M Rogers, Aaron Roth, and Steven Z Wu. 2016. Learning from rational behavior: Predicting solutions to unknown linear programs. In Advances in Neural Information Processing Systems. 1570-1578.

[16] Dmytro Korzhyk, Vincent Conitzer, and Ronald Parr. 2010. Complexity of Computing Optimal Stackelberg Strategies in Security Resource Allocation Games.. In AAAI.

[17] Brian Kulis et al. 2013. Metric learning: A survey. Foundations and Trends ${ }^{\circledR}$ in Machine Learning 5, 4 (2013), $287-364$.

[18] Wei Liu and Sanjay Chawla. 2009. A game theoretical model for adversarial learning. In Data Mining Workshops, 2009. ICDMW'09. IEEE International Conference on. IEEE, 25-30.

[19] Ralph Tyrell Rockafellar. 1970. Convex analysis. Princeton University Press.

[20] Aaron Roth, Aleksandrs Slivkins, Jonathan Ullman, and Zhiwei Steven Wu. 2017. Multidimensional Dynamic Pricing for Welfare Maximization. In Proceedings of the 2017 ACM Conference on Economics and Computation. ACM, 519-536.

[21] Aaron Roth, Jonathan Ullman, and Zhiwei Steven Wu. 2016. Watch and learn: Optimizing from revealed preferences feedback. In Proceedings of the forty-eighth annual ACM symposium on Theory of Computing. ACM, 949-962.

[22] Milind Tambe. 2011. Security and game theory: algorithms, deployed systems, lessons learned. Cambridge University Press.

[23] Morteza Zadimoghaddam and Aaron Roth. 2012. Efficiently Learning from Revealed Preference.. In WINE. Springer, 114-127.

[24] Martin Zinkevich. 2003. Online convex programming and generalized infinitesimal gradient ascent. In Proceedings of the 20th International Conference on Machine Learning (ICML-03). 928-936. 